\title{
Silica-Supported Proline Derivatives for Catalytic Studies
}

\author{
Tatiane Inforzato ${ }^{1}$, Liane Marcia Rossi ${ }^{2}$, Tiago Venancio, ${ }^{1}$ Alcindo A. Dos Santos ${ }^{2}$ \\ ${ }^{1}$ Departamento de Química, Universidade Federal de São Carlos, Rod. Washington Luis Km 235 - CEP \\ 13565-905, São Carlos - SP. ${ }^{2}$ Instituto de Química, Universidade de São Paulo, Av. Prof. Lineu Prestes, 748 \\ - CEP 05509-000, São Paulo - SP \\ *e-mail tatianeinforzato@yahoo.com.br:
}

Keywords: proline derivatives, hybrid organo-inorganic materials, catalysis

\section{INTRODUCTION}

Heterogenization of organic catalysts would be an effective way to improve their catalytic efficiency via catalyst recovery and reuse. Efforts have been devoted to the immobilization and recycling of Lproline ${ }^{1}$. In this work, we present the detention of prolinamide and prolinethioamide in silica. These materials can be applied in catalytic reactions.

\section{RESULTS AND DISCUSSION}

Initially, for the functionalization of the silica surface with proline we prepared compound $\mathbf{1}$ which was treated with ethyl chloroformate followed by N-Bocproline to afford 2 according Scheme 1. The Boc group was removed by acid treatment, allowing the preparation of the hybrid organo-inorganic material 2. All intermediates as well the target material $\mathbf{2}$, were submitted to characterization analysis, including ${ }^{13} \mathrm{C}-\mathrm{NMR} / \mathrm{CPMAS}$. Material 2 was also submitted to microanalysis and was found that the functionalization resulted in $0.46 \mathrm{mmol}$ of organic residue/g of silica. Compound 2 was converted into the corresponding thioamide by treatment with Lawesson's reagent. Additionally, compound 1 was converted into $\mathbf{4}$ which will be submitted to reaction with prolinamide as well prolinethioamide to prepare the corresponding thioureas.

Scheme 1. Funcionalization of the prolinamide and prolinethiomide.
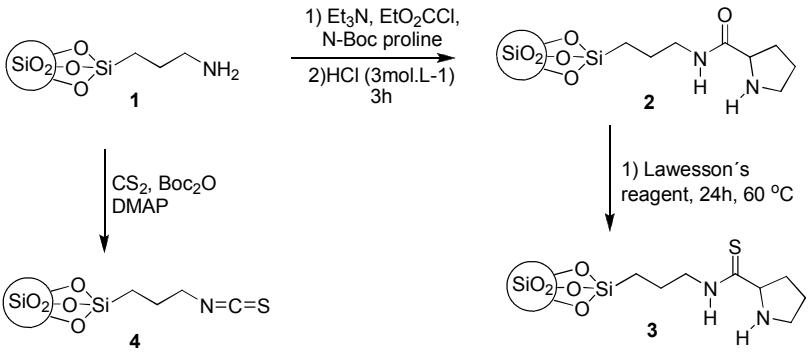

Figure 1 presents the designed organo-inorganic hybrid materials that will be prepared from $\mathbf{4}$ or $\mathbf{5}$.

Figure 1. Ureas and Thioureas that will be prepared

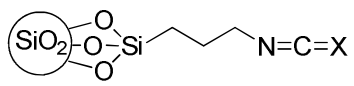

4, $\mathrm{X}=\mathrm{S}$ or $5, \mathrm{X}=\mathrm{O}$

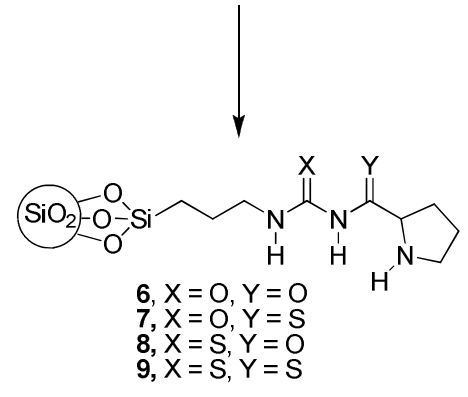

Next, these materials will be teasted as catalysts for Michael addition, Strecker and Mannich reactions.

\section{CONCLUSION}

In conclusion, we demonstrated the preparation of hybrid organo-inorganic materials by efficient procedures. The new material $\mathbf{3}$ was produced to be used as catalyst in order to investigate the performance compared with $\mathbf{2}$. Tests catalysis using this material is already being carried out in our laboratory.

\section{ACKNOWLEDGEMENTS}

CNPq, FAPESP and CAPES for financial support.

\section{REFERENCES}

${ }^{1}$ Gruttadauria,M.; Giacalone,F.; Noto, R. Chem. Soc. Rev. 2008,37,1666.

Materials 1-4 were submitted to $I R,{ }^{13} \mathrm{C}$ NMR/CPMAS, ${ }^{29} \mathrm{Si}-\mathrm{NMR}$ and microanalysis and are in agreement with the proposed structures. 